\title{
An impaired phagocytic function is associated with leucocyte activation in the early stages of severe acute pancreatitis
}

\author{
G Liras, F Carballo
}

\begin{abstract}
Background-An excessive leucocyte activation takes place early in severe acute pancreatitis. Furthermore, some indirect evidences suggest a disturbance of the mononuclear phagocytic system in the severe cases.

Aims-To compare the early functionalism of leucocytes obtained from patients with mild disease and severe disease, under the hypothesis that an impaired phagocytic function could be associated with the leucocyte activation.

Patients and methods-Flow cytometric parameters of leucocyte function, such as phagocytosis, and fluorescence of leucocytes with acridine orange (FLAO) were prospectively measured together with granulocyte elastase plasma concentrations in 21 patients with severe $(n=7)$ and mild $(n=14)$ acute pancreatitis. Samples were drawn at 24 hours from admission, 48 hours, 72 hours, and on day 5.

Results-There was a greater leucocyte activation together with a deficient phagocytosis before 72 hours in severe patients. The results (mean (SEM)) severe $v$ mild were: 440 (115) $\mu g / 1 v 77$ (14) $\mu g / 1$ for granulocyte elastase, 2.218 (377) $v 1.308$ (155) for FLAO, 64 (7) $v 90$ (2), and 55 (9) $v 81$ (3) for percentages of phagocytising neutrophils and monocytes, respectively. Phagocytic capacity returned to normal later on day 5.

Conclusions-The excessive leucocyte activation together with the impaired phagocytosis could be related to the onset of complications in severe acute pancreatitis.
\end{abstract}

(Gut 1996; 39: 39-42)

Keywords: acute pancreatitis, phagocytosis, neutrophils, flow cytometry, pancreatopeptidase, multiple organ failure.

Research Unit and Department of Internal Medicine, University Hospital, Guadalajara and INSALUD, University of Alcalá de Henares, Spain

G Liras

F Carballo

Correspondence to: Dr F Carballo, Unidad de Investigación, Hospital Universitario de Guadalajara Universitario de Guadalajara 19002 Guadalajara, Spain.

Accepted for publication

13 February 1996

Activated neutrophils and macrophages play a central part in the pathophysiology of severe acute pancreatitis. ${ }^{1-3}$ Neutrophil activation is triggered by stimulating adhesion molecules exposed in the damaged endothelial cells. ${ }^{4}$ Activated polymorphonuclear granulocytes release potent lysosomal proteases, mainly granulocyte elastase, together with oxygen free radicals. ${ }^{5}$ These oxidising agents inactivate several protease inhibitor systems such as $\alpha_{1}$ antiprotease or $\alpha_{2}$ macroglobulin. ${ }^{6}$ So, free proteases can exert their harmful effects not only in a local environment but also spreading further the damage and activating proteolytic systems as complement, kinin, coagulation, and fibrinolysis cascades. ${ }^{7-9}$ This complex mechanism has been implicated in the development of both local (that is, infected necrosis, abscess, pseudocyst) and systemic complications of severe pancreatitis, as multiple organ failure, adult respiratory distress syndrome or disseminated intravascular coagulation. ${ }^{10}$ On the other hand, the mononuclear phagocytic system participates in various reparative processes, such as clearance of bacteria, cellular debres, and protease-antiprotease complexes. Some clinical and experimental indirect evidence suggest an impaired functionalism of the mononuclear phagocytic system in complicated acute pancreatitis. ${ }^{1112}$ In this study the basis for the hypothesis was that the impaired phagocytic functionalism could be associated with the leucocyte activation in severe cases. To confirm this hypothesis we prospectively measured by flow cytometry the early phagocytic activity of leucocytes and its time course in peripheral blood samples obtained from patients with acute pancreatitis. Additionally, we studied other parameters of leucocyte function, such as cellular volume, and nuclear activation measuring the fluorescence of vitally stained samples with acridine orange (FLAO). Moreover, we simultaneously analysed the plasma concentration of leucocyte proteases as an index of neutrophil activation, and as indicator of the leucocyte mediators release into the inflammatory focus. We show new data supporting the hypothesis that activated neutrophils together with a deficient phagocytic functionalism could be related to the development of complications in patients with severe acute pancreatitis.

\section{Methods}

\section{Patients}

A total of 21 patients with acute pancreatitis were prospectively included in the study. Diagnosis of acute pancreatitis was based on characteristic clinical symptoms and increased serum activities of amylase, at least twice the upper limit of the reference range (20-128 U/1). All patients underwent subsequent investigation by imaging techniques (ultrasonography and computed tomography) to confirm the diagnosis and to clarify the aetiology of the episode. The mean (SD) age of the patients was $66.4(13.7)$ years; two were male and 19 were female. By aetiology, there was a 
Number of major complications developed in patients with severe acute pancreatitis. Balthazar grade of computed tomogram is also shown

\begin{tabular}{|c|c|c|c|c|c|c|c|}
\hline Patient & 1 & 2 & 3 & 4 & 5 & 6 & 7 \\
\hline $\begin{array}{l}\text { Balthazar grade (CT) } \\
\text { Necrosis }\end{array}$ & $\underset{\star}{E}$ & $\underset{\star}{\mathrm{D}}$ & D & C & $\underset{\star}{\mathrm{D}}$ & D & $\underset{\star}{D}$ \\
\hline Necrosis and haemorrhage & & & * & $\star$ & & & \\
\hline Pseudocyst & & & & & & * & \\
\hline Cardiac insufficiency & & & 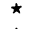 & * & & & \\
\hline Respiratory insufficiency & & * & * & * & * & & * \\
\hline Renal failure & & $\star$ & * & * & * & & \\
\hline Shock & & * & * & * & & & \\
\hline Sepsis & & & & & $\star$ & $\star$ & \\
\hline Fatal outcome & & & + & + & & & \\
\hline
\end{tabular}

pronounced predominance of biliary pancreatitis (17 patients), and not one of the patients presented alcoholic pancreatitis. Four patients presented idiopathic pancreatitis. Fifteen patients presented a first episode, while six were relapses. The interval between the onset of symptoms and admission of the patient to the hospital was short - that is, two to 12 hours.

Patients were classified into two groups, mild pancreatitis $(n=14)$ and severe pancreatitis $(n=7)$, taking into account the findings of the computed tomography (CT), and the development of one or more systemic complications of the disease (Table). The criteria for diagnosing local and systemic complications were those generally accepted. ${ }^{13}$ The information obtained in the CT was classified according to Balthazar et al. ${ }^{14}$ No patients of the group with mild disease showed a score of the CT analysis equal of upper than grade C, according to Balthazar classification.

Scores of severity according to the Imrie classification $^{15}$ were also obtained. In the group of mild disease the mean (SD) of Imrie criteria was $1.5(0.9)$ and the interval of values ranged between 0 and 3. Three patients of this group fulfilled three Imrie criteria. In the group of severe disease the mean (SD) was $4 \cdot 6(1 \cdot 1)$ and the interval of values, 3 to 6 . Of all patients, seven required surgery (six cholecystectomy and one drainage of pseudocyst).

Among patients with severe disease one (patient 1 , Table) presented with a persistent leucocytosis, impaired coagulation laboratory tests, raised granulocyte elastase $(601 \mu \mathrm{g} / \mathrm{l})$ and fever, although blood cultures were negatives. Two patients (2 and 3, Table) needed intensive care treatment as a result of their respiratory insufficiency and acute renal failure. Of these two, one died at day 4 as a consequence of multiple organ failure development. Another patient with severe pancreatitis (patient 4, Table) also died as a consequence of multiple organ failure development at day 13 after admission. Two patients (5 and 6, Table) developed sepsis by Escherichia coli and Staphylococcus aureus methycillin resistance, respectively, and one also presented a pseudocyst that required surgery. The last patient included in this group suffered respiratory insufficiency at admission.

Laboratory methods

EDTA and heparin anticoagulated venous blood samples were drawn at the first 24 hours from the admission, 48 hours, 72 hours, and on day 5 of hospitalisation. EDTA anticoagulated plasma samples were frozen at $-70^{\circ} \mathrm{C}$ and subsequently analysed for granulocyte elastase concentrations using the immunoactivation assay (Merck, Darmstadt, Germany) and following the specifications of the manufacturer. Reference range (mean value (SD)) of the assay is $22(10) \mu \mathrm{g} / \mathrm{l}$. Flow cytometric analysis were performed immediately (before two hours) in heparinised $(10 \mathrm{U} / \mathrm{ml})$ blood samples obtained from 21 patients. Samples from 12 healthy donors were also analysed to attain an adequate performance of the cytometric procedures. The percentage of phagocytising cells (neutrophils and monocytes) was assayed in whole heparinised blood samples with the Phagotest kit (Orpegen, Heidelberg, Germany) measuring fluorescence of 5000 leucocytes per sample in a FACScan flow cytometer (Becton Dickinson, San Jose, CA) and following the procedure indicated by the manufacturer. The expected interval of values for phagocytosis in healthy normal subjects is $70-90 \%$ for monocytes and $70-95 \%$ for neutrophils.

Fluorescent staining of buffy coat leucocytes obtained by sedimentation at $200 \mathrm{~g}$ and $4^{\circ} \mathrm{C}$ for 10 minutes was performed following the procedure of Rothe et al. ${ }^{16}$ The leucocytes were collected at the erythrocyte/plasma interface and resuspended in autologous plasma at a final concentration of $3.5 \times 10^{7}$ leucocytes/ $\mathrm{ml}$. This cell suspension $(250 \mu \mathrm{l})$ was incubated for 15 minutes at $4^{\circ} \mathrm{C}$ with $5 \mu$ of a dye cocktail containing $0.96 \mathrm{mmol} / 1$ of acridine orange (Sigma) and $3 \mathrm{mmol} / \mathrm{l}$ of propidium iodide (Becton Dickinson) in N,N-dimethylformamide (Sigma). Acridine orange stains the DNA of leucocytes and propidium iodide counterstains the DNA of dead leucocytes. The fluorescence of 3000 cells per sample was measured in the FACScan cytometer exciting with an argon laser light $(488 \mathrm{~nm})$ and measuring fluorescence at $530 \mathrm{~nm}$ (acridine orange green fluorescence) and at $575 \mathrm{~nm}$ (acridine orange and propidium iodide red fluorescence). Cellular volume of leucocytes was calculated by measuring the forward angle light scattering of leucocytes as previously reported. ${ }^{1718}$ The flow cytometer was calibrated with the CaliBRITE flow cytometer beads reagent (Becton Dickinson, San Jose, CA).

\section{Statistical methods}

Comparison of data between both groups of patients was performed by the Mann-Whitney test. Coefficient of correlation and statistical significance between values of granulocyte elastase and fluorescence intensity with acridine orange were also calculated.

\section{Results}

Figure 1 shows the plasma granulocyte elastase values obtained in the patients' samples. Statistical significance was found between both groups of patients during the time course studied. Figures 2 and 3 show the percentage 


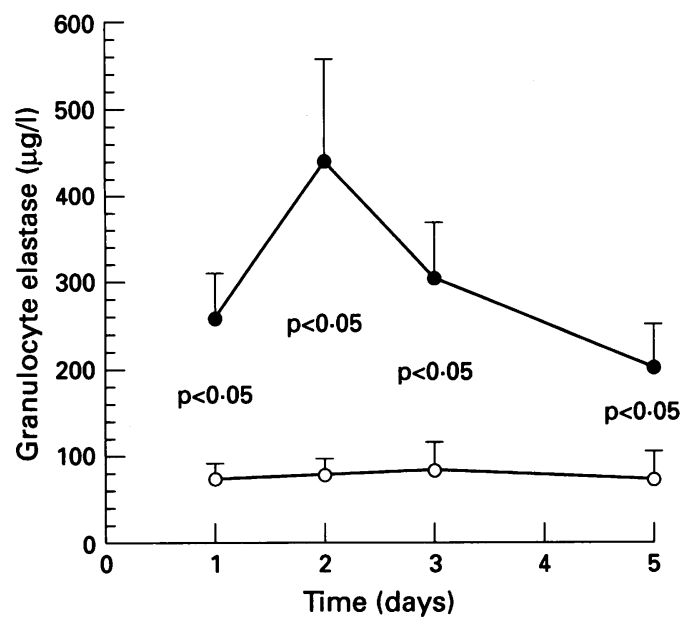

Figure 1: Plasma granulocyte elastase values (mean (SEM)) obtained in patients with mild disease (O) and severe disease (๑). Statistical significance is shown. of active phagocytic cells (neutrophils and monocytes), cellular volume of leucocytes, and fluorescence intensity values (arbitrary units) with acridine orange. Before 72 hours a lower phagocytic capacity for neutrophils and monocytes were found in severe pancreatitis compared with mild pancreatitis. However, granulocyte elastase activities and fluorescence with acridine orange were greater in patients with severe disease, indicating an excessive neutrophil activation. On day 5, only fluorescence with acridine orange and granulocyte elastase activities remained increased in severe patients. The leucocyte activation expressed by the fluorescence intensity with acridine orange was directly related to the granulocyte elastase plasma values, with a coefficient correlation of $0.5474(p<0.05)$. With regard to cellular volume of leucocytes, statistical significance was not achieved in the comparison between both groups of patients.

\section{Discussion}

We have simultaneously measured by flow cytometry phagocytic and functional activities of leucocytes together with granulocyte elastase release in patients suffering mild and severe episodes of acute pancreatitis. Our findings are in agreement with the data obtained by Rothe et al. ${ }^{16}$ These authors found systemic changes in neutrophil function (phagocytosis, intracellular $\mathrm{pH}$, cellular volume) associated with the development of post-traumatic or septic multiple organ failure in critically ill patients. Further indirect evidence of an impaired functionalism of the mononuclear phagocytic system in severe acute pancreatitis has been found by other authors. ${ }^{11}$ By example, a pronounced depletion in serum $\alpha_{2}$ macroglobulin concentrations in severe pancreatitis has been noted. ${ }^{19} 20$ An adequate macrophage functionalism is vital to remove cellular debris, bacteria, and the most important, perform the clearance of protease-antiprotease complexes. If this clearance is not adequately performed these complexes could further spread systemically the damage. Different responses of postsurgical macrophages to IL1 have been found ${ }^{21}$ measuring secretion of protease and protease inhibitor activities after in vitro exposure to IL1. This different behaviour of macrophages was found at various postsurgical times. In our study, an impaired phagocytic response was shown mainly in the early stages of the disease ( $<72$ hours), and on day 5 the phagocytic capacity of leucocytes was unable to differentiate between patients with severe and mild acute pancreatitis. Despite this fact, a deficient phagocytosis could be found later in septic patients, as was found in the patient with sepsis that developed a pseudocyst. However, in the other patient with sepsis an early deficient phagocytic response was demonstrated. It would therefore be interesting to further investigate the phagocytic capacity of leucocytes with a greater number of patients suffering from acute pancreatitis and septic complications.
Figure 3: Fluorescence intensity with acridine orange and cellular volume of leucocytes in samples obtained before 72 hours and later on day 5. Mean (SEM) are shown for patients with mild disease (O) and severe disease ( $\bullet$ ). Statistical significance is shown (ns, not significant). Values obtained in the group of healthy donors are represented by continuous lines (mean) and discontinuous lines (SEM). 
Another remarkable finding concerning phagocytosis was that this parameter normalised in the two patients suffering imminent multiple organ failure and fatal outcome. Therefore, the percentage of active phagocytic cells cannot be used in the prognosis of an imminent severe complication of the illness. Nevertheless, this finding is in accordance with previous reports ${ }^{16}$ and controversy exists about the origin of this recovered functionalism. One explanation offered is based on the recruitment of neutrophils from the marginated blood vessel cell pool or from the bone marrow cell pool, ${ }^{16}$ although hyperergic phagocytosis could also be a consequence of the neutrophil activation by anaphylotoxins - that is, C3a and C5a complement cleavage products. ${ }^{22}$

With regard to the results obtained measuring the fluorescence with acridine orange and granulocyte elastase values, we confirmed the previous findings obtained by other authors 162023 and also by our group. ${ }^{24}$ The greater vital staining of resting neutrophils with acridine orange is related to changes of chromatin condensation and nuclear activation. ${ }^{25}$ It is interesting to note that fluorescence intensity with acridine orange and granulocyte elastase activities remained increased even on day 5 in the group with severe disease. Data shown in Figures 1 and 3 show that leucocytes obtained from patients with severe disease were more activated and that such activation remained for a longer time in the early course of the disease. However, phagocytic capacity was recovered on day 5 , and therefore clearance of protease-antiprotease complexes could be adequately performed, as was also confirmed by the decrease of granulocyte elastase plasma concentrations. This fact could indicate a persistence of leucocyte activation, which is present together with a greater clearance of protease-antiprotease complexes, at this time point. Furthermore, fluorescence intensity with acridine orange decreased more in the group of patients with mild disease than in the group of patients with severe pancreatitis.

An increased leucocyte cellular volume has been also related to the process of activation. ${ }^{1618}$ In our study, greater mean values were found in patients with severe disease, and also in the early stages of the disease, but statistical significance could not be attained in the comparison between both groups of patients. However, it is important to note that cellular volume measured by the forward angle light scattering can also be affected by other parameters (not only the process of activation), as the internal structure of the cell and the refractive indices of cells and medium. ${ }^{18}$

Data obtained strongly support the evidence that an impaired functionalism of phagocytes is present together with an excessive activation of leucocytes in the early phases of severe acute pancreatitis. Nevertheless, it would be interesting in the near future to extend this study to a series with a greater number of acute necrotising pancreatitis or severe systemic complications, or both, such as the adult respiratory distress syndrome, the intravascular disseminated coagulation, and septic complications.

This study was supported by Health Investigation Fund (FIS) grant number $94 / 5014$

1 Rinderknecht $\mathrm{H}$. Fatal pancreatitis, a consequence of excesive leucocyte stimulation? Int $f$ Pancreatol 1988; 3 sive leuco $105-12$.

2 Gross V, Andreesen R, Leser HG, Ceska M, Liehl E, Lausen $M$, et al. Interleukin-8 and neutrophil activation in acute pancreatitis. Eur f Clin Invest 1992; 22: 200-3.

3 Sanfey H, Bulkley GB, Cameron JL. The role of oxygenderived free radicals in the pathogenesis of acute pancreatitis. Ann Surg 1984; 200: 405-13.

4 Nathan CF. Neutrophil activation on biological surfaces: massive secretion of hydrogen peroxide in response to products of macrophages and lymphocytes. $\mathcal{f}$ Clin Invest 1987; 80: 1550-60.

5 Henson PM, Johnston RB. Tissue injury in inflammation: oxidants, proteinases, and cationic proteins. $7 \mathrm{Clin}$ Invest 1987; 79: 669-74.

6 Weiss SJ, Curnutte JT, Regiani S. Neutrophil-mediated solubilization of the subendothelial matrix:oxidative and nonoxidative mechanisms of proteolysis used by norma and chronic granulomatous disease phagocytes. $\mathcal{F}$ Immunol 1986; 136: 636-41.

7 Lasson A, Ohlsson K. Acute pancreatitis. The correlation between clinical course, protease inhibitors and complement and kinin activation. Scand $\mathcal{F}$ Gastroenterol $1984 ; 19$ $707-10$.

8 Orlov V, Belyakov N. Blood kallikrein-kinin system in acute pancreatitis. Am $\mathcal{F}$ Gastroenterol 1978; 70: 645-8.

9 Lasson A, Ohlsson K. Consumptive coagulopathy, fibrinolysis and protease-antiprotease interactions during fibrinolysis and protease-antiprotease interactions during

10 Redl H, Schlag G. Pathophysiology of multi-organ failure (MOF) proposed mechanisms. Clinical Intensive Care 1990; 1: 66-71.

11 Browder IW, Sherwood E, Williams D, Jones E, McNamee R, Diluzio N. Protective effect of glucan-enhanced macrophage function in experimental pancreatitis. $A m \mathcal{F}$ Surg 1987; 153: 25-33.

12 Adham NF, Song MK, Haberfelde GC. Relationship between the functional status of the reticuloendothelial system and the outcome of experimentally induced pancreatitis in young mice. Gastroenterology 1983; 84: 461-9

13 Glazer G. Classification and definitions in acute and chronic pancreatitis. In: Glazer G, Ranson JHC, eds. Acute pancreatitis. Experimental and clinical aspects of pathogenesis and management. London: Baillière Tindall, 1988 $37-50$.

14 Balthazar EJ, Ranson JHC, Naidich DP, Megibow AJ, Caccavale R, Cooper MM. Acute pancreatitis: prognostic value of CT. Radiology 1985; 156: 767-72.

15 Blamey SL, Imrie CW, O'Neill J, Gilmour WH, Carter DC Prognostic factors in acute pancreatitis. Gut 1984; 25: 1340-6.

16 Rothe G, Kellermann W, Valet G. Flow cytometric parameters of neutrophil function as early indicators of sepsis- or trauma-related pulmonary or cardiovascular organ failure. trauma-related pulmonary or card

17 Mullaney PF, Dean PN. The small angle light scattering of biological cells. Theoretical considerations. Biophys $f$ 1970; 10: 764-72.

18 Grinstein S, Furuya W, Cragoe EJ Jr. Volume changes in activated human neutrophils: the role of $\mathrm{Na}^{+} / \mathrm{H}^{+}$ exchange. f Cell Physiol 1986; 128: 33-40.

19 Goodman AJ, Bird NC, Johnson AG. Antiprotease capacity in acute pancreatitis. Br $\mathcal{F}$ Surg 1986; 73: 796-8

20 Gross V, Schölmerich J, Leser HG, Salm R, Lausen M, Rückauer $\mathrm{K}$, et al. Granulocyte elastase in assessment of severity of acute pancreatitis. Dig Dis Sci 1990; 35: 97-105.

21 Kuraoka S, Campeau JD, Rodgers KE, Nakamura RM, diZerega GS. Effects of interleukin-1 (IL-1) on postsurgical macrophage secretion of protease and protease cal macrophage secretion of protease and
inhibitor activities. $\mathcal{F}$ Surg Res $1992 ; 52: 71-8$.

22 O'Flaherty JTO, Kreutzer DL, Ward PA. Chemotactic factor influences on the aggregation, swelling and foreign surface adhesiveness of human leucocytes. Am $\mathcal{f}$ Pathol 1978; 90: 537-50.

23 Uhl W, Büchler M, Malfertheiner P, Martini M, Beger HG PMN-Elastase in comparison with CRP, antiproteases, and $\mathrm{LDH}$ as indicators of necrosis in human acute pancreatitis. Pancreas 1991; 6: 253-9.

24 Domínguez-Muñoz JE, Carballo F, García MJ, de Diego JM, Rábago L, Simón MA, et al. Clinical usefulness of polymorphonuclear elastase in predicting the severity of cute pancreatitis: results of a multicentre study. $\mathrm{Br} F$ Surg 1991; 78: 1230-4

25 Traganos F, Darzynkiewicz Z, Sharpless T, Melamed MR Cytofluorometric studies on conformation of nucleic acids in situ. I Restriction chromatin proteins. $\mathcal{f}$ Histochem Cytochem 1976; 24: 40-8. 